\title{
Determination of cardiac output by Doppler echocardiography
}

\author{
HALFDAN IHLEN, JAN P AMLIE, JON DALE, KOLBJØRN FORFANG, SIGURD \\ NITTER-HAUGE, JAN E OTTERSTAD, SVEIN SIMONSEN, ERIK MYHRE \\ From the Medical Department B, Rikshopitalet, University Hospital, Oslo, Norway
}

SUMmaRY Cardiac output determined by Doppler echocardiography was compared with that determined by thermodilution at rest and during dobutamine infusion in 10 patients (group $A$ ) and by the Fick method at rest in 11 patients (group B). All patients had angina pectoris without valvular heart disease. Maximum spatial blood velocity and cross sectional aortic area were estimated by the Doppler technique and echocardiography. Cardiac output was calculated by multiplying blood velocity by aortic area at various levels in the ascending aorta. The best correlation of cardiac output between the invasive and non-invasive methods was obtained when maximum velocity in the aortic root and the aortic orifice area were used in the calculations. Cardiac output was considerably overestimated when area measurements in the aortic root were used.

The Doppler technique for measuring blood velocities in the human aorta was first reported by Light. ${ }^{1}$ Later several investigators attempted to estimate cardiac output by multiplying the velocity by the cross sectional area of the aorta,,$^{2-4}$ but the results have been conflicting. ${ }^{5-7}$ One important factor is that there is no general agreement on where in the aorta the area or the blood velocity should be measured. The present investigation was, therefore, designed to elucidate this problem by comparing the results of the non-invasive method with those of two generally accepted invasive methods-thermodilution and the Fick method.

\section{Patients and methods}

\section{STUDY POPULATION}

Twenty one patients ( 18 men, three women, mean age 53 (range 41-64) years with angina pectoris who were considered for coronary bypass surgery gave their informed consent to participate in the study. In this hospital the haemodynamic evaluation of angina pectoris includes complete left and right heart catheterisation. Nineteen patients had coronary heart disease, one cardiomyopathy, and one no evidence of disease. All had regular sinus rhythm and were without valvular heart disease or intracardiac shunts. No other measurements of their fitness for ultrasonography were carried out. For practical reasons, the patients

Accepted for publication 30 August 1983 were examined either in the ultrasound laboratory (group A) or in the catheterisation laboratory (group B).

\section{Group A}

This group included 10 patients: eight with moderate angina pectoris, one with cardiomyopathy, and one without known heart disease. In this group, dimensions in the aortic root were measured in detail, and dobutamine hydrochloride was given to increase cardiac output. The dobutamine hydrochloride was infused with an initial dose of $2.5 \mu \mathrm{g} / \mathrm{kg} / \mathrm{min}$ which was increased to 5.0 (eventually 10.0 ) $\mu \mathrm{g} / \mathrm{kg} / \mathrm{min}$ if well tolerated. Cardiac output was measured with a thermodilution catheter (Balltherm thermal dilution catheter, Elecath). Velocity measurements by the Doppler technique were carried out simultaneously with estimations of cardiac output at rest and during the highest dobutamine dose. Thus 20 estimates of cardiac output were obtained in this group.

\section{Group B}

This group included the remaining 11 patients, all with angina pectoris which was considered to be too severe to be tested with positive inotropic drugs. Velocity measurements in these patients were performed in the catheterisation laboratory at the end of the routine right heart catheterisation. Cardiac output was simultaneously measured by the Fick method. 
MATHEMATICAL CONSIDERATIONS

Volumetric flow rate $(Q)$ in the aorta may be calculated as: $Q(t)=\bar{v}(t) \times A$ where $\bar{v}$ is the spatial mean blood velocity, $A$ is the area of the cross section of the vessel, and $t$ indicates that the variables are functions of time.

Using the Doppler technique, the spatial mean velocity may be obtained from the mean Doppler shift if the artery is uniformly insonated. The narrow ultrasound beam, however, insonates only a part of the cross section of the artery. Animal experiments have shown that the velocity profile in the aorta is fairly flat, ${ }^{8}$ an observation which is likely from mathematical considerations since we have accelerated flow in a large artery. For a flat velocity profile the spatial mean velocity will be close to the spatial maximum of the velocity in the centre of the lumen. The maximum velocity measured by the narrow Doppler beam, therefore, approximates the spatial mean velocity. Assuming that forward flow in the ascending aorta occurs only during systole, and the angle between the ultrasonic beam and the direction of flow is less than $25^{\circ}, 9$ the traversed distance of blood may be obtained by integrating the velocity measured over systole. The cross sectional area may be measured by echocardiography. Assuming a circular area of the ascending aorta unaffected by systole, the cross sectional area (A) may be calculated as $\mathbf{A}=$ $\left(\frac{\mathrm{D}}{2}\right)^{2} \times \pi$ where $\mathrm{D}$ is the diameter. Cardiac output (CO) may then be obtained using the equation $\mathrm{CO}=$ SVI $\times A \times$ HR where SVI is the systolic velocity integral, $A$ the area, and HR the heart rate.

\section{DOPPLER TECHNIQUE}

The Doppler recordings were carried out with two nearly identical instruments. In the ultrasound laboratory we used a commercially available multifrequency (1-10 MHz) Doppler instrument (ALFRED, Vingmed) which may be used in either a pulsed or a continuous mode. With the pulsed mode the sample volume is a cylinder with an approximate diameter of $8 \mathrm{~mm}$ and a length of $5 \mathrm{~mm}$. The received signals are fed to an audio amplifier and are also processed by two frequency estimators which convert them into analogue voltages proportional to the mean and maximum frequency shifts. These estimators determine mean and maximum velocities accurately in experimental studies and in the human aorta (Fig.1).1011 An integrator automatically calculates the velocity integral and is reset at the beginning of each heart cycle by an electrocardiographic signal. As maximum velocities are less affected by errors in aiming than mean velocities, ${ }^{12}$ we decided to integrate the maximum velocity curve. The velocity curves, the amplitude of the Doppler signal, and the maximum velocity integral were recorded on a Mingograph 82 elec-

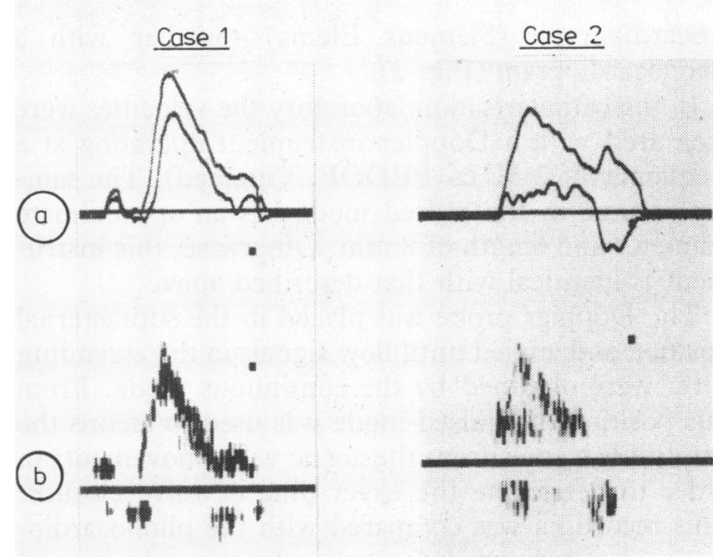

Fig. 1 Simultaneous analysis of maximum and mean velocities using pulsed Doppler signals by (a) analogue estimators and (b) spectral analysator (Chirp-Z Fourier transform) from aortic root with normal valves (calibration equal). Note that optimal signals are present in case 1 showing similar results when maximum and mean velocities are determined by the two outputs. The recording of maximum velocity is also correct in case 2. Nevertheless, $a$ broad spectrum of velocities are present, probably owing to incorrect aiming of the Doppler beam, which affects mean velocity more than maximum velocity.

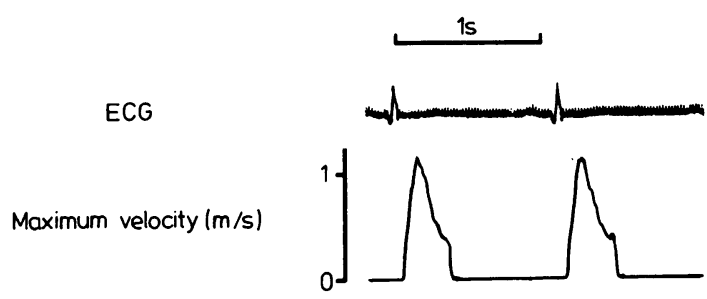

Phonocardiogram

Mean velocity $(\mathrm{m} / \mathrm{s})$

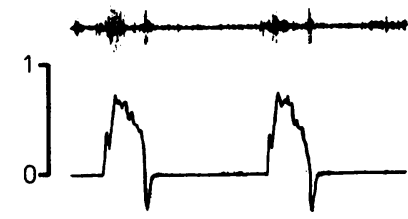

Amplitude of Doppler signal

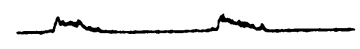

Integral of maximum velocity $(\mathrm{cm})$

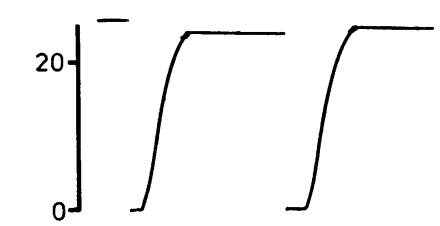

Fig. 2 Electrocardiogram (ECG), phonocardiogram, and analogue signals obtained from the Doppler system showing maximum and mean velocities and maximum velocity integral. 
trocardiograph (Siemens Elema) together with a phonocardiogram (Fig. 2).

In the catheterisation laboratory the velocities were measured with a Doppler instrument operating at a frequency of $2 \mathrm{MHz}$ (PEDOF, Vingmed). The sample volume in the pulsed mode has an approximate diameter and length of $8 \mathrm{~mm}$. Otherwise, this instrument is identical with that described above.

The Doppler probe was placed in the suprasternal position and angled until flow signals in the ascending aorta were obtained by the continuous mode. From this position the pulsed mode was used to record the amplitude signals from the aortic valve movements in order to determine the exact time of valve closure. This recording was compared with the phonocardiogram recorded simultaneously which was later used for identification of this timing.

Velocity recordings were obtained $(a)$ from the level immediately above the valve, $(b)$ from a position $2 \mathrm{~cm}$ higher in the aorta, and $(c)$ in the ascending aorta by the continuous mode. Before the measurements, the beam direction was adjusted to obtain the highest velocities, and we confirmed that the gain setting of the ultrasound was safely below the setting at which noise interference occurs. Care was taken to ensure that valve movements did not enter the Doppler sample volume. The criteria for acceptance of adequate signals ${ }^{9}$ were $(a)$ a high frequency whistling sound when listening to the audible signal and $(b)$ a smooth velocity curve characterised by a rapid increase to peak velocity during the early phase of systole. The maximum velocity was integrated automatically through each systole. The end of these integrals was taken at the closure of the aortic valve. All calculations were based on the mean of 10 consecutive beats.

\section{ECHOCARDIOGRAPHY}

Echocardiograms were recorded using an IREX III phased array cross sectional instrument and the aortic dimensions measured by a digitising computer system (CARDIO 80, Kontron). This system allowed measurements to be taken directly from the video picture by an electronic cursor controlled by movement of a pen on a measuring tablet.

In group $\mathrm{A}$, the aortic diameters obtained by cross sectional echocardiography in the parasternal long axis view were measured at four different sites (Fig.3): (a) high in the left ventricular outflow tract, $(b)$ at the aortic orifice, $(c)$ in the proximal aortic root with valve echoes by a derived $M$ mode line, and $(d)$ in the distal aortic root $2 \mathrm{~cm}$ above the orifice. All diameters were measured from the leading edge of the anterior echo to the leading edge of the posterior echo and compared with the trailing edge to leading edge standard. ${ }^{1314}$ Care was taken to ensure that the dimensions were measured at the midpoint of the
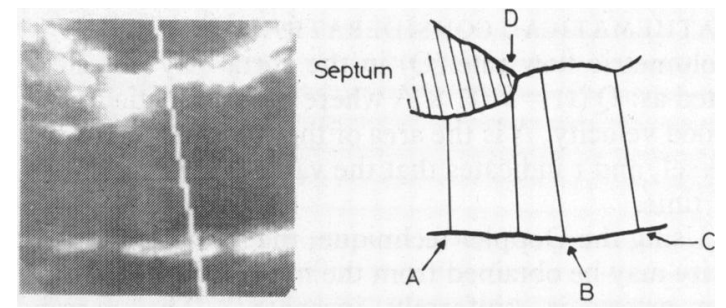

Fig. 3 Cross sectional echocardiogram in the long axis view of the aorta and aortic valve region with the different sites of diameter measurement: $A$, left ventricular outflow tract; $B$, proximal aortic root (derived $M$ mode line); $C$, distal aortic root; $D$, aortic orifice.

lumen perpendicular to the luminary long axis. Diastolic and systolic diameters were measured at the $Q$ wave and the peak of the $T$ wave on the electrocardiogram respectively, and the arithmetical mean of the two values was used for area calculations. The anterior wall echo of the orifice was defined as the echo from the highest portion of the septum under the fast moving valve structures. The distance from this echo to the posterior wall, measured perpendicular to the long axis of the aortic root, was the diameter of the orifice. The proximal aortic root area at the level of the valves was also recorded directly from a short axis parasternal view using the black/white echocardiographic interface of the lumen. ${ }^{13}$ In group $B$, diameters were measured at the level of the aortic orifice and $2 \mathrm{~cm}$ above.

Before catheterisation all patients were examined by echocardiography. In group $\mathrm{A}$, the echocardiograms were repeated during the measurements of cardiac output at rest and after dobutamine.

\section{INVASIVE TECHNIQUES}

An Elecath Cardiac Output Computer 4000 with digital display was used for the thermodilution measurement of cardiac output, the temperature curves being recorded on a Mingograph 34 electrocardiograph. The output estimate was calculated from three consecutive measurements with identical temperature curves. With this technique, the variation about the mean of the three measurements is $<5 \%$ in our laboratory. The variation in results between the thermodilution and the Fick methods is $<15 \%$. Oxygen content in air and blood was determined using an ABL 3 and OSM 2 apparatus (Radiometer, Copenhagen), respectively.

\section{STATISTICAL ANALYSIS}

Non-invasive and invasive measurements were made by different investigators, and the results were not compared until the study was completed. Statistical analysis was performed using Wilcoxon's rank sum test (on paired data) and linear regression analysis. The 
Table 1 Integrals of the systolic maximum velocity (cm) recorded by the Doppler technique in groups $A$ and $B$ (mean of 10 beats). Figures are means \pm 1 standard deviation.

\begin{tabular}{|c|c|c|c|}
\hline & \multicolumn{2}{|c|}{ Pulsed mode techrique } & \multirow{2}{*}{$\begin{array}{l}\text { Continuous mode } \\
\text { technique }\end{array}$} \\
\hline & Aortic root & $\begin{array}{l}2 \mathrm{~cm} \text { above } \\
\text { aortic valves }\end{array}$ & \\
\hline $\begin{array}{l}\text { Group } \\
\text { A }(n=20) \\
\text { Group } \\
B(n=11)\end{array}$ & $\begin{array}{l}23 \cdot 3 \pm 4 \cdot 2 \\
15 \cdot 6 \pm 3 \cdot 8(n=10)\end{array}$ & $\begin{array}{l}23 \cdot 1 \pm 4 \cdot 1(n=19) \\
15 \cdot 0 \pm 3 \cdot 6(n=8)\end{array}$ & $\begin{array}{l}22 \cdot 1 \pm 3 \cdot 8(n=16) \\
17 \cdot 4 \pm 5 \cdot 8(n=5)\end{array}$ \\
\hline
\end{tabular}

$\mathrm{n}$, number of measurements.

values are expressed as means \pm 1 standard deviation (SD).

\section{Results}

\section{DOPPLER MEASUREMENTS}

Technically adequate maximum velocity curves were obtained at the level of the valve in all but one recording. From a position $2 \mathrm{~cm}$ above the valve level, four recordings were inadequate and 10 using the continuous mode had to be excluded. The velocity integrals measured by the three recording techniques were equal in both groups (Table 1).

\section{Aortic area}

Technically acceptable recordings were obtained in all 21 patients from the cross sectional long axis view. Diameters measured from the leading edge of the anterior wall to the leading edge of the posterior wall were generally $1-2 \mathrm{~mm}$ greater than those measured from the trailing edge to the leading edge. Only diameters obtained using the leading edge to leading edge standard are used in the calculations. In group $A$, the measured areas were identical at all levels in the two control periods and after giving dobutamine (Table 2).

The proximal aortic root area measured from the cross sectional short axis view as well as its diameter recorded by a derived $M$ mode line showed a significant increase of $14 \%$ and $8 \%$ during systole. The diameters of the outflow tract, the aortic orifice, and the distal aortic root did not change significantly from diastole to systole. The diastolic areas were: (a) Outflow tract $3.6 \pm 1.2 \mathrm{~cm}^{2}$, (b) aortic orifice $5.0 \pm 1.2$ $\mathrm{cm}^{2}$, (c) proximal aortic root area $8.1 \pm 1.6 \mathrm{~cm}^{2}$ from the cross sectional short axis view and $8.5 \pm 1.5 \mathrm{~cm}^{2}$ from the derived $M$ mode line, and (d) distal aortic root area $7.7 \pm 0.8 \mathrm{~cm}^{2}$.

\section{Cardiac output}

In general the non-invasive Doppler measurements showed a considerable overestimation compared with the invasive results (Table 3). Cardiac output was on average $80 \%$ higher by the non-invasive than invasive techniques when the Doppler velocity integral and cross sectional area of the proximal aortic root were used in our calculations (in group $A$ ). The noninvasive output estimate was lower when recordings in the distal aortic root were used, but was $56 \%$ and $54 \%$ higher than the invasive estimate in groups $A$ and $B$ respectively. Non-invasive measurements also were an overestimation compared with the invasive data, when the continuous mode integral was multiplied by the distal aortic root area, and were $49 \%$ and $102 \%$ higher than corresponding invasive values in groups $\mathrm{A}$ and $B$ respectively.

Nevertheless, when the proximal aortic root integral was multiplied by the aortic orifice area, cardiac output paralleled that by the invasive method in group $\mathrm{A}$ and was slightly lower than that by the oxygen method in group B. Individual Doppler data correlated closely with the invasive technique data in group A $(r=0.96)$ and in group B ( $r=0.90)$ (Fig. 4). Using this technique cardiac output could be determined non-invasively in all but one patient $(95 \%)$ in group B. During dobutamine infusion heart rate increased insignificantly from a mean of 57 to 61 beats/min. An increase in the maximum velocity integral was the main reason for the increased non-

Table 2 Aortic root area and diameters in ouflow tract and ascending aorta in group A (10 patients) during two control periods and dobutamine infusion. Figures are means $\pm I S D$

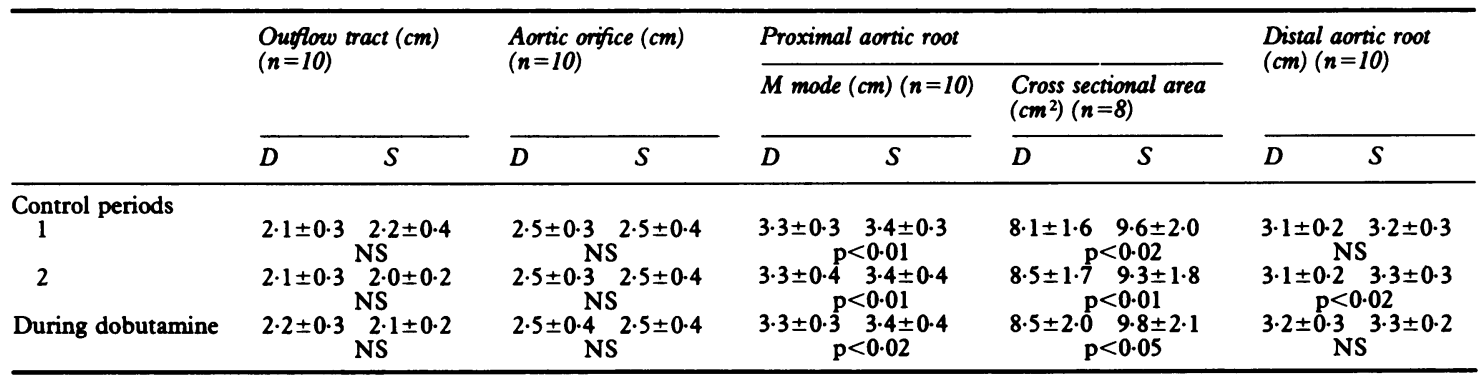

$\mathrm{D}$, diastole; $\mathrm{S}$, systole; $\mathrm{n}$, number of measurements. 
Table 3 Cardiac output (l/min) determined by Doppler ultrasound $(U)$ and invasive $(I)$ techniques. Figures are means $\pm S D$

\begin{tabular}{|c|c|c|c|c|}
\hline & Proximal aortic root & Distal aortic root & & Aortic orifice \\
\hline & $U$ & $U$ & $U t$ & $U$ \\
\hline $\begin{array}{l}\text { Group A }(n=20) \\
\text { Group B }(n=11)\end{array}$ & $\begin{array}{c}12 \cdot 2 \pm 0 \cdot 9 * \quad 6 \cdot 8 \pm 2 \cdot 1 \\
(n=20)\end{array}$ & $\begin{array}{c}10 \cdot 6 \pm 2.9 * \quad 6 \cdot 6 \pm 2 \cdot 0 \\
(n=19) \\
7 \cdot 9 \pm 1 \cdot 9 * \quad 5 \cdot 1 \pm 1 \cdot 5 \\
(n=7)\end{array}$ & $\begin{array}{c}10 \cdot 4 \pm 3 \cdot 1^{\star} \quad 7 \cdot 0 \pm 1 \cdot 9 \\
(n=15) \\
8.9 \pm 2 \cdot 5^{\star} \quad 4 \cdot 4 \pm 1 \cdot 3 \\
(n=5)\end{array}$ & $\begin{array}{c}6 \cdot 6 \pm 1 \cdot 8 \quad 6 \cdot 8 \pm 2 \cdot 1 \\
(n=20) \\
4 \cdot 4 \pm 1 \cdot 5 \star \star \\
(n=10)\end{array}$ \\
\hline
\end{tabular}

$\mathrm{n}$, number of measurements.

${ }^{\star} p<0.01$ versus invasive methods; $\star \star p<0.02$ versus invasive methods.

tContinuous mode Doppler.

invasive cardiac output estimates after dobutamine infusion (Fig. 4).

\section{Discussion}

The Doppler instrument used in this study measures maximum velocity by a frequency estimator which determines maximum velocity accurately in experimental studies. ${ }^{11}$ The velocity estimator is different from the zero crossing detector used in some instruments. While it is generally accepted that spectral analysis of the Doppler signal should be used for detecting luminal stenosis, measurements of flow in the human aorta without valvular disease have shown close correlations between maximum velocity derived from a complete spectral analysis and that calculated by the estimator. 11

An incorrect angle between the ultrasound beam and the direction of blood flow is the major problem when blood velocities are measured by the Doppler technique. Combined instruments visualise the sample volume of the pulsed Doppler in the echocardiographic picture, and the angle between the Doppler beam and the blood flow may be determined in two planes. This technique measures cardiac output exactly when applied to children, ${ }^{6}$ whereas the results have not been so good in adults. ${ }^{4}$ The integral of the systolic mean velocity measured centrally in the vessel was used in both studies. Our measurements were performed without knowing the exact site of the sample volume. Low frequency signals from aortic walls or adjacent vessels may interfere with the measurements of the mean velocity, 1215 and errors in aiming of the ultrasound beam may result in underestimating flow measurements (Fig. 1). The maximum velocity is, however, derived exclusively from the aortic flow ${ }^{9}$ and was therefore used in this study.

Satisfactory curves of the maximum velocity were obtained easily by the pulsed mode Doppler technique. We consider that the pulsed technique is preferable, since maximum velocity from the continuous mode provides velocity information from all along the Doppler beam. A highly complex velocity spectrum is recorded, and the highest velocity may be obtained at a site other than the aortic root. We measured maximum velocity satisfactorily in only $65 \%$ of the continuous mode recordings. On the other hand, Huntsman et al. using the continuous mode technique and spectral analysis estimated cardiac output in a large number of patients. ${ }^{7}$

Mathematical considerations imply that velocity and aortic area should be measured at the same level since velocity has to change to maintain the same vol-
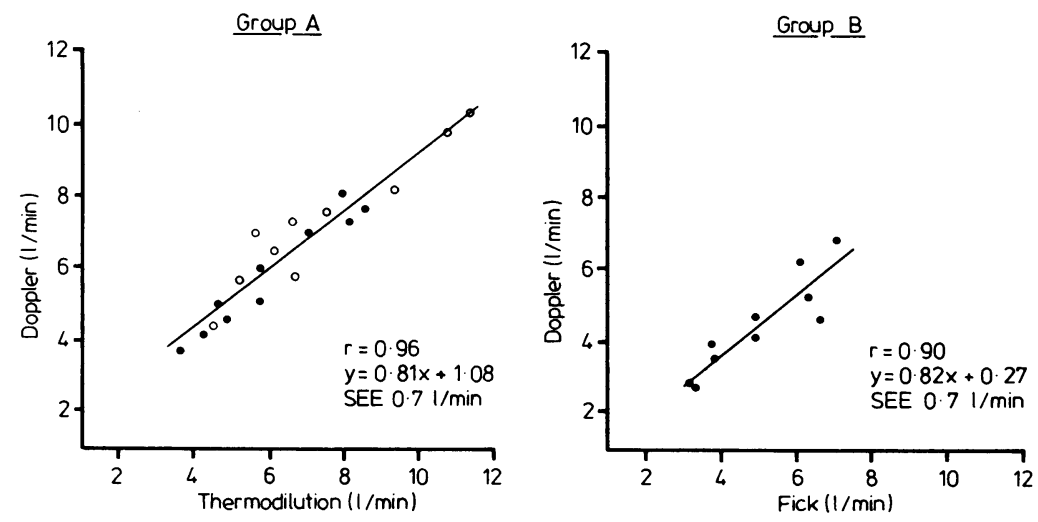

Fig. 4 Linear regression analysis of cardiac output obtained by combining Doppler measurements with thermodilution in group $A$ and with the Fick method in group B. Non-invasive cardiac output is estimated as the maximum velocity integral in the proximal aortic root $\times$ aortic orifice area $\times$ heart rate. $(\bullet)-$ at rest; ( $(0)$ - during dobutamine infusion. 
ume flow with the altered cross sectional area. Thus measurement of the cross sectional aortic area may be the prime source of error in estimating cardiac output by ultrasonography. ${ }^{616} \mathrm{We}$ measured the area by several echocardiographic techniques. Calculation of the aortic area from the diameter assumes a circular shape, which is not present in pure form in man, and errors in diameter measurements are squared. These problems are avoided by using the cross sectional short axis technique, which provides a direct measurement of the aortic root area. The problem with this technique is that the lateral resolution is notably less good than the axial resolution. Most workers today agree that identification of the sites for area measurement requires cross sectional echocardiography, although aortic diameters are easier to measure from the $M$ mode echocardiogram than from the cross sectional echo. The level at which aortic valve echoes are seen is, however, probably the only reproducible site for cross sectional short axis and single $M$ mode recordings. We find that the two latter techniques did not improve the reproducibility of the area measurements. The same conclusion has also been reached by others. ${ }^{17}$ Another problem in estimating the area is that the aortic root area increases during systole owing to the elastic properties of the aortic wall. In our study this increase was about $10 \%$, which is consistent with other reports. ${ }^{18}$ The only site which was unchanged by systole was the aortic orifice, and its diameter was also more reproducible than the aortic root diameters. Recently another group of workers had similar results. ${ }^{14}$

We measured diameters from the leading edge of the anterior echo to the leading edge of the posterior echo. Mathematically, this method has been assumed to be the most accurate. ${ }^{13}$ Huntsman et al. determined the aortic root diameter by the A mode technique using the trailing edge to leading edge standard. ${ }^{7}$ They measured the diameter in the region immediately above the sinuses of Valsalva and found correct estimates of cardiac output when this was multiplied by the maximum velocity integral. The diameter used in our study measured $2 \mathrm{~cm}$ above the aortic orifice was probably obtained at approximately the same level as that in their study. ${ }^{7}$ Our diameter measurements, however, would have been reduced by 1-2 $\mathrm{mm}$ if the trailing edge to leading edge standard had been used. The mean aortic diameter $2 \mathrm{~cm}$ above the aortic orifice thus would have been about $3.0 \mathrm{~cm}$ which is exactly the same as that found by Huntsman et al. ${ }^{7}$ Our results are, therefore, similar to those of others and confirm difficulties in determining aortic root diameters.

In the present study, we found that area measurements varied at different sites within the aorta, whereas identical maximum velocities were obtained at the three sampling positions. Our findings, which are in agreement with those of a recent report, ${ }^{16}$ suggest that the sampling site for blood velocities is of less importance, whereas the level at which the area is measured influences estimates of cardiac output considerably. When the highly reproducible measurements of aortic orifice area were combined with those of maximum velocity, we obtained a good agreement between invasive and non-invasive estimates of cardiac output. On the other hand, the cardiac output was considerably overestimated when the area and velocity were measured in the proximal as well as in the distal aortic root.

Our findings contrast with those of other workers who have shown that optimal results are obtained when measurements of velocity and cross sectional area dimensions in the aortic root are used. ${ }^{367}$ The errors in determining aortic area were too small to explain the overestimation ( $49 \%$ to $102 \%)$ of cardiac output obtained in our study. It is unlikely that our Doppler instrument measures higher velocities than other instruments, but we may have obtained a smaller angle between the Doppler beam and the blood flow than other workers. An overestimation of the spatial mean blood velocity in the aortic root owing to disturbed blood flow may also explain our findings. As far as we know there are no detailed data on the velocity distribution within the human aortic root. Nevertheless, mathematical considerations ${ }^{9}$ and ani-

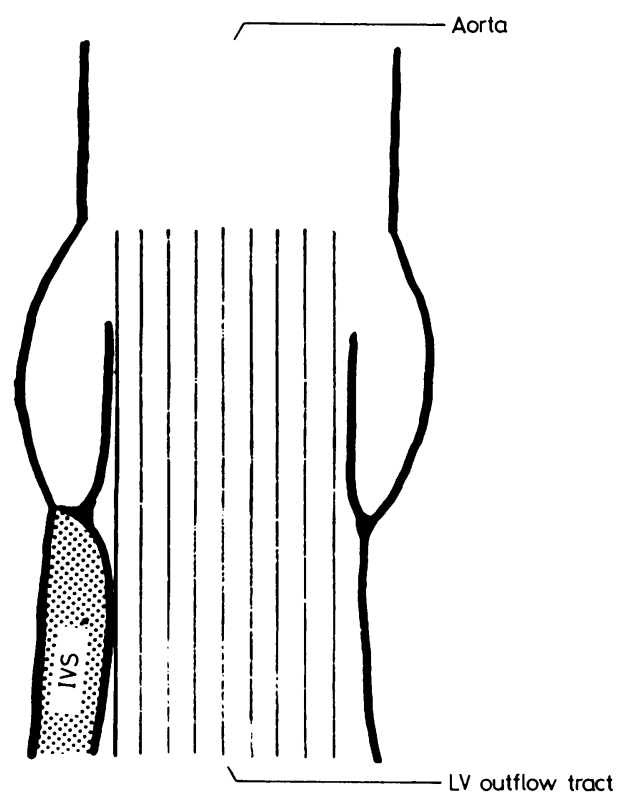

Fig. 5 Theory of "plug" formed flow from left ventricular $(L V)$ ouffow tract into the aortic root. IVS, interventricular septum. 
mal experiments ${ }^{8}$ strongly suggest laminar blood flow with a flat spatial velocity profile, which was a prerequisite for this study. The echocardiographic data in our patients show that blood is ejected through the outflow tract and the aortic orifice into a appreciably larger aortic root. The effective area over which blood flow occurs in the aortic root may be considerably smaller than the anatomical aortic root area. The results of the present study, therefore, suggest indirectly that there is a "plug" formed flow out of the left ventricle with a cross sectional flow area determined by the aortic orifice (Fig. 5).

In conclusion, our results show that determinations of cardiac output in adults should be calculated from the aortic orifice area and maximum velocity in the aortic root. When the aortic root area is used in the calculations, cardiac output may be considerably overestimated.

\section{References}

1 Light LH. Transcutaneous observations of blood velocity in the ascending aorta in man. $\mathcal{F}$ Physiol (Lond) 1969; 204: 1P-2P.

2 Sequeira RF, Light LH, Cross G, Raftery EB. Transcutaneous aortovelography. A quantitative evaluation. $\mathrm{Br}$ Heart $\mathcal{F}$ 1976; 38: 443-50.

3 Chandraratna PAN, Nanna M, McKay C, Nimalasuriya A, Sweeney R, Ramhimtoola SH. Determination of cardiac output by transcutaneous continuous wave ultrasonic Doppler computer [Abstract]. Circulation 1982; 66 (suppl II):II-121.

4 Magnin PA, Stewart JA, Myers S, Von Ramm O, Kisslo JA. Combined Doppler and phased-array echocardiographic estimation of cardiac output. Circulation 1981; 63: 388-92.

5 Waters J, Kwan OL, Kerns G, et al. Limitations of Doppler echocardiography in the calculation of cardiac output [Abstract]. Circulation 1982; 66 (suppl II):II-122.

6 Goldberg SJ, Sahn DJ, Allen HD, Valdes-Cruz LM, Hoenecke H, Carnahan Y. Evaluation of pulmonary and systemic blood flow by 2-dimensional Doppler echocar- diography using fast Fourier transform spectral analysis. Am F Cardiol 1982; 50: 1394-400.

7 Huntsman LL, Stewart DK, Barnes SR, Franklin SB, Colocousis JS, Hessel EA. Noninvasive Doppler determination of cardiac output in man. Clinical validation. Circulation 1983; 67: 593-602.

8 Seed WA, Wood NB. Velocity patterns in the aorta. Cardiovasc Res 1971; 5: 319-30.

9 Hatle L, Angelsen B. Doppler ultrasound in cardiology. Philadelphia: Lea \& Febiger, 1982: 70-5.

10 Angelsen BAJ, Brubakk AO. Transcutaneous measurement of blood flow velocity in the human aorta. Cardiovasc Res 1976; 10: 368-79.

11 Brubakk AO, Angelsen BAJ, Hatle L. Diagnosis of valvular heart disease using transcutaneous Doppler ultrasound. Cardiovasc Res 1977; 11: 461-9.

12 Brubakk AO, Givold SE. Pulsed Doppler ultrasound for measuring blood flow in the human aorta. In: Hatle L, Angelsen B, ed. Doppler ultrasound in cardiology. Philadelphia: Lea \& Febiger, 1982: 185-93.

13 Feigenbaum H. Echocardiography. 3rd ed. Philadelphia: Lea \& Febiger, 1981: 110-5.

14 Gussenhoven WJ, Van Leenen BF, Kuis W, De Villeneuve VH, Bon N, Van Meurs-van Woezik H. Comparison of internal diameter of great arteries in congenital heart disease. A cross sectional echocardiographic study. Br Heart f 1983; 49: 45-50.

15 Steingart RM, Meller J, Barovick J, Patterson R, Herman MV, Teichholz LE. Pulsed Doppler echocardiographic measurement of beat-to-beat changes in stroke volume in dogs. Circulation 1980; 62: 542-8.

16 Fisher DC, Sahn DJ, Friedman MJ, et al. The effect of variations on pulsed Doppler sampling site on calculation of cardiac output: an experimental study in open-chest dogs. Circulation 1983; 67: 370-6.

17 Gardin JM, Tobis JM, Dabestani A, et al. Superiority of two-dimensional measurement of aortic vessel diameter in Doppler echocardiographic estimates of left ventricular stroke volume [Abstract]. Clin Res 1983; 31: 8A.

18 Greenfield JC Jr, Patel DJ. Relation between pressure and diameter in the ascending aorta of man. Circ Res 1962; 10: 778-81.

Requests for reprints to Dr Halfdan Ihlen, Medical Department B, Rikshospitalet, Oslo 1, Norway. 